\title{
Is the Concurrent Use of Sorafenib and External Radiotherapy Feasible for Advanced Hepatocellular Carcinoma? A Meta-Analysis
}

\author{
Chai Hong Rim ${ }^{1, *,+}$, Sunmin Park ${ }^{1,+} \mathbb{\infty}$, In-Soo Shin ${ }^{2}$ and Won Sup Yoon ${ }^{1}$ \\ 1 Department of Radiation Oncology, Ansan Hospital, Korea University Medical College, Ansan 15355, Korea; \\ sunmini815@gmail.com (S.P.); irionyws@korea.ac.kr (W.S.Y.) \\ 2 Graduate School of Education, Dongguk University, Seoul 04620, Korea; 9065031@hanmail.net \\ * Correspondence: crusion@korea.ac.kr or crusion3@naver.com; Tel.: +82-31-412-6850; Fax: +82-31-412-6851 \\ + These authors contributed equally to this work.
}

Citation: Rim, C.H.; Park, S.; Shin, I.-S.; Yoon, W.S. Is the Concurrent Use of Sorafenib and External Radiotherapy Feasible for Advanced Hepatocellular Carcinoma? A Meta-Analysis. Cancers 2021, 13, 2912. https://doi.org/10.3390/

cancers 13122912

Academic Editor: Hidehito Horinouchi

Received: 19 May 2021

Accepted: 9 June 2021

Published: 10 June 2021

Publisher's Note: MDPI stays neutral with regard to jurisdictional claims in published maps and institutional affiliations.

Copyright: (c) 2021 by the authors Licensee MDPI, Basel, Switzerland. This article is an open access article distributed under the terms and conditions of the Creative Commons Attribution (CC BY) license (https:/ / creativecommons.org/licenses/by/ $4.0 /)$
Simple Summary: Concurrent chemotherapy and external radiation is a commonly used method for cancer treatment. However, concurrent application of sorafenib and external radiotherapy has not been commonly used in clinical practice due to the possible risk of excessive complication. The results of this meta-analysis suggest that concurrent treatment might be a feasible option, and treatment targeting metastatic lesion or vessel involvement is particularly recommended.

Abstract: We evaluate the feasibility of a concurrent application of sorafenib and external beam radiation therapy (EBRT) for advanced hepatocellular carcinoma (HCC). PubMed, Embase, Medline, and Cochrane Library were searched up to 9 April 2021. The primary endpoint was grade $\geq 3$ complications, and the secondary endpoint was overall survival (OS). Subgroup analyses were performed for studies with the EBRT targets, intrahepatic vs. non-intrahepatic lesions (e.g., extrahepatic metastases or malignant vessel involvement only). Eleven studies involving 512 patients were included in this meta-analysis. Pooled rates of gastrointestinal, hepatologic, hematologic, and dermatologic grade $\geq 3$ toxicities were $8.1 \%$ (95\% confidence interval $\left.(\mathrm{CI}): 4.8-13.5, \mathrm{I}^{2}=\sim 0 \%\right), 12.9 \%(95 \% \mathrm{CI}$ : $7.1-22.1, \mathrm{I}^{2}=22.4 \%$ ), 9.1\% (95\% CI: $3.8-20.3, \mathrm{I}^{2}=51.3 \%$ ), and $6.8 \%$ (95\% CI: $3.8-11.7, \mathrm{I}^{2}=\sim 0 \%$ ), respectively. Pooled grade $\geq 3$ hepatologic and hematologic toxicity rates were lower in studies targeting non-intrahepatic lesions than those targeting intrahepatic lesions (hepatologic: $3.3 \%$ vs. $17.1 \%, p=0.041$; hematologic: $3.3 \%$ vs. $16.0 \%, p=0.078$ ). Gastrointestinal and dermatologic grade $\geq 3$ complications were not significantly different between the subgroups. Regarding OS, concurrent treatment was more beneficial than non-concurrent treatment (odds ratio: 3.3, 95\% CI: 1.3-8.59, $p=0.015)$. One study reported a case of lethal toxicity due to tumor rupture and gastrointestinal bleeding. Concurrent treatment can be considered and applied to target metastatic lesions or local vessel involvement. Intrahepatic lesions should be treated cautiously by considering the target size and hepatic reserve.

Keywords: sorafenib; external beam radiation therapy; concurrent; combined; hepatocellular carcinoma; toxicity

\section{Introduction}

Hepatocellular carcinoma (HCC) has a tendency to rapidly change the tumor microenvironment and metabolic profile, resulting in tumorigenic and proliferation properties [1] Although surveillance programs have been conducted in many countries, many patients are still diagnosed with advanced disease. Adherence to surveillance is less than 50\% [2] and updated modalities could not be applied to all populations at high risk, although sonography still has a major role [3-5]. From a wider perspective, approximately $70 \%$ of patients were unresectable at diagnosis in China [6]; one-third of patients were found 
to have Barcelona Clinic of Liver Cancer (BCLC) C or higher disease in Korean National Database data [7].

External beam radiation therapy (EBRT) and chemotherapy have been used in combination to treat diverse cancers. The basic principle of this treatment includes spatial cooperation, where radiation targets macroscopic diseases, and the systemic agent treats latent microscopic disease. In addition, chemotherapy increases the effectiveness of EBRT by inducing cell cycle arrest and interfering with DNA damage repair [8,9]. Sorafenib has been established as a systemic agent that can increase the survival of patients with advanced HCC [10]. EBRT has also recently been shown to have significant effects on HCC with major vessel involvement or metastases [11-13]. Preclinical evidence regarding the synergistic effect of sorafenib and EBRT has been reported [14,15]. Nevertheless, few clinical studies have examined the concurrent use of sorafenib and radiotherapy. This scarcity is due to concerns about excessive toxicities [16-18].

Brade et al. [16] reported a serious hepatotoxicity rate of approximately $20 \%$, which highlights the risk of concomitant therapy with EBRT and sorafenib. However, several researchers [19-21] have reported their experience with such combination therapy, and the degree of serious toxicities was not significantly different from that reported in the previous relevant literature (e.g., sequential treatment). In addition, as modern radiation therapy (RT) modalities such as intensity-modulated radiotherapy (IMRT) and stereotactic body radiotherapy (SBRT) become common, the targets of EBRT are becoming smaller, and consequently, the risk of toxicity caused by RT has been decreasing [22,23].

Although the concurrent treatment of sorafenib and EBRT can have synergistic benefits, clinical application is rare due to the risk of toxicity reported by a small number of previous studies. Therefore, the risks and effectiveness of concurrent applications are mostly unknown. This meta-analysis aimed to compile published data to gain a better understanding of the feasibility of using a combined treatment in advanced HCC.

\section{Methods}

\subsection{Searching Process}

We adhered to the PRISMA guidelines [24] to conduct the present systematic review and meta-analysis as well as we could, and referred to the Cochrane handbook version 6.2 for methodological direction [25]. The present study was designed to answer the following clinical PICO question: "Is the concurrent application of sorafenib and EBRT a feasible option (as compared to sequential or non-combined modalities) for patients with advanced HCC?" The studies that met the following inclusion criteria were included: (1) clinical trials involving the application of concurrent sorafenib and EBRT; (2) those including at least five patients with HCC who underwent such concurrent treatment; and (3) those with data regarding primary and/or secondary endpoints. We searched databases such as PubMed, Embase, Medline, and Cochrane Library, as recommended by the Cochrane handbook [26], for literature until 9 April 2021. The search term was designed to identify studies related to combined or concurrent use of sorafenib and EBRT for liver cancers, and the detailed search strategy is described in Supplementary Document 1. Language restrictions were not included. Chinese articles were translated by a professional Chinese-English translator, whereas Japanese or Korean articles were translated by one of the authors (CHR). Reference lists of related articles were also searched. Conference abstracts were considered if they met the inclusion criteria and were exhibited in established conferences (e.g., including but not limited to ASCO, ASTRO, AASLD, EASL, ESTRO, ESMO, and RANZCR). All searching and inclusion processes were performed by two independent researchers, and any disagreement was resolved by mutual discussion and repetition of the search. 


\subsection{Data Items and Collection}

The primary endpoint of the present study was grade $\geq 3$ complications and the secondary endpoint was overall survival (OS). We used standardized sheets to collect the following data: (1) general information including names of authors, year of publication, affiliation, conflicts of interests, year of patient recruitment, number of patients; (2) clinical information including target disease, rate of Child-Pugh class A, EBRT modality and dose, the target of EBRT, sorafenib dose; (3) outcomes of interest including rates of grade $\geq 3$ gastrointestinal, hepatologic, hematologic, and dermatologic complications, grade 4 or 5 complications, radiation-induced liver disease (RILD), and OS. Data extraction processes were performed by two independent researchers, and any disagreement was resolved by re-evaluation of the literature and discussion.

\subsection{Risk of Bias and Assessment of the Quality}

Because the application of concurrent sorafenib and EBRT has never been assessed in randomized trials, the possible risk of bias was discussed based on the Cochrane handbook chapter for the assessment of non-randomized studies [27]. The primary outcome of interest was treatment toxicity. Considering possible subjectivity in the assessment of treatment toxicities, and to facilitate comprehensive quantitative synthesis, we evaluated the possible toxicities by using four clinical categories (gastrointestinal, hepatologic, hematologic, and dermatologic). In addition, descriptions of complications in the studies were qualitatively analyzed. Regarding pooled analyses of OS, odds ratios between comparative arms (concurrent treatment vs. radiation therapy (RT) without sorafenib or sorafenib without RT) were synthesized, rather than pooled analyses of OS percentile rates, considering the diversity of target disease among studies. Because the searched studies were mostly observational studies, the Newcastle-Ottawa scale [28] was used for quality assessment.

\subsection{Statistics}

The principal summary measures were percentile rates of categorized grade $\geq 3$ complications. OS was also pooled and analyzed, which yielded an odds ratio, using a median survival or 1-year OS rate. Median survival periods were estimated as mean values as necessary, using the method suggested by Hozo et al. [29]. The random effects model was used for pooled analyses of primary endpoints because the majority of studies were observational studies; this was in accordance with the recommendation in the Cochrane handbook that the random effects model should be the default model for analyzing nonrandomized studies [30]. Heterogeneity assessment was performed using the Cochran $\mathrm{Q}$ [31] test and $\mathrm{I}^{2}$ statistics [32]; significant heterogeneity was considered to be present when a $p$-value of $<0.1$ and $\mathrm{I}^{2}>50 \%$ were obtained. Publication bias was assessed using visual inspection of funnel plots and the quantitative Egger's test [33] for pooled analyses involving 10 studies. Possible publication bias was deemed to present with visual asymmetry in funnel plot analysis and a two-tailed $p$-value of $<0.1$ in Egger's test. Grade 4-5 complications, RILD, and OS were also qualitatively evaluated. Subgroup analyses were performed for studies focusing on intrahepatic versus non-intrahepatic lesions (e.g., denote targeting extrahepatic metastases or vessel involvement only), as local targets of EBRT, and a $p$-value below 0.1 denotes a statistically significant subgroup difference [34]. All statistical analyses were performed using Comprehensive Meta-Analysis version 3 (Biostat Inc., Englewood, NJ, USA). 


\subsection{Protocol Registration}

This study is registered in PROSPERO (ID: CRD42021248705).

\section{Results}

\subsection{Study Selection and Characteristics}

Among the 961 initially identified studies, we excluded 586 studies owing to irrelevant formats and 22 studies owing to duplication among databases. The abstract screening was performed on 295 studies, and a full-text review was performed on 47 studies after excluding 248 studies with irrelevant subjects or formats. A full-text review was performed to identify studies that fully met the inclusion criteria, and 11 studies were finally included in the present study [16,18-21,35-40]. The selection process is illustrated in Figure 1.
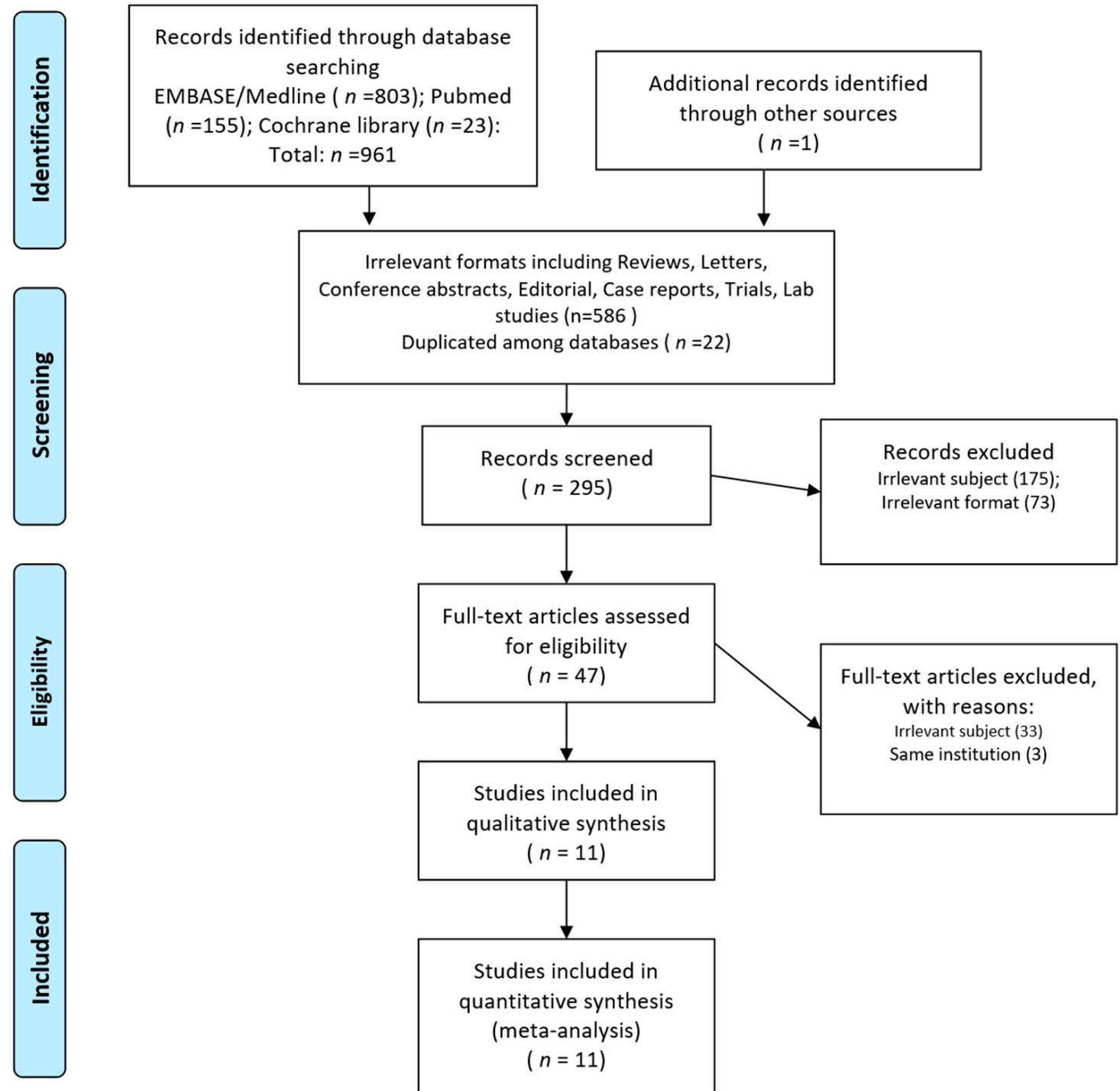

Figure 1. Study inclusion process. 
Among the included studies, eight were from China or Taiwan, and one each was from Canada, Japan, and Korea. Nine studies were published as full-text articles, and two were abstracts published by the American Society for Radiation Oncology (ASTRO). All are written in English except one study written in Chinese [35]. The earliest study recruited patients from 2006 to 2009 and the latest from 2007 to 2017. Six studies were designed to have comparative arms (e.g., those with patients who underwent concurrent treatment vs. treatments involving EBRT without sorafenib or sorafenib without EBRT), and five were single-arm studies involving patients who received concurrent treatment. Rates of Child-Pugh A ranged from 65.6\% to 100\% (median 100\%). Regarding RT modality, IMRT or tomotherapy was performed in five studies, SBRT in two studies, conventional 3dimensional conformal RT in two studies, and brachytherapy in two studies. The majority of studies prescribed sorafenib in doses of $400 \mathrm{mg}$ bid and were modified considering toxicities. The general characteristics of the included studies are summarized in Table 1.

\subsection{Quality Assessment and Selection of Studies}

Regarding quality assessment using NOS [28], four queries in the selection category were mostly fulfilled by all studies; because patients' characteristics were representative of the population, outcomes of interest (e.g., complications or survival) were not present at the beginning of the study, and treatment modalities were well defined. Regarding queries in the outcome category, follow-up was adequate in the majority of studies included because they evaluated survival or toxicity for a sufficient period in patients with relatively short expected survival. Regarding comparability, we allotted two points (full point) for studies involving comparative arms without significant differences in clinical characteristics, or those that used propensity matching methods. These studies were considered to have reliable comparability. One point was allotted to studies involving comparative arms, but without statistical comparison. Single-arm studies were allotted zero points. Overall, five comparative studies were allotted nine points, one comparative study was allotted eight points, and five single-arm studies were allotted seven points. The detailed results are shown in Table S1.

\subsection{Summary of Individual Study Results}

The rate of grade $\geq 3$ gastrointestinal, hepatologic, hematologic, and dermatologic complication ranged from $0 \%$ to $20 \%, 0 \%$ to $20 \%, 0 \%$ to $33.3 \%$, and $0 \%$ to $16.7 \%$, respectively. The median value of the median survival period of patients who underwent concurrent application of sorafenib and RT was 17.4 months (range: 7.8-31.2). Among six studies with comparative design $[20,21,35,38-40]$, none of the studies reported significant differences in grade $\geq 3$ toxicity between arms, in quantitative and qualitative terms. Regarding OS comparison, four of six studies $[20,21,35,39]$ reported that the OS benefit of concurrent treatment was significantly higher than that of non-concurrent treatment; one study reported a non-significant trend [40], and one study reported no difference [38]. The clinical results of the studies are shown in Table 2. 
Table 1. Study characteristics.

\begin{tabular}{|c|c|c|c|c|c|c|c|c|c|c|c|}
\hline Author & $\begin{array}{c}\text { Publication, } \\
\text { Year }\end{array}$ & Affiliation & $\begin{array}{c}\text { Recruitment, } \\
\text { Year }\end{array}$ & Study Design & $\begin{array}{c}\text { Target } \\
\text { Disease }\end{array}$ & $\begin{array}{c}\text { No. of } \\
\text { Patients * }\end{array}$ & СРС A (\%) & Sorafenib & RT Modality & Dose & $\begin{array}{c}\text { Other } \\
\text { Combination } \\
\text { Tx. }\end{array}$ \\
\hline Sun [20] & $\begin{array}{l}\text { Oncotarget, } \\
2016\end{array}$ & $\begin{array}{l}\text { Fudan Univ, } \\
\text { China }\end{array}$ & 2011-2014 & Comparative & $\begin{array}{c}\text { Lung } \\
\text { metastases }\end{array}$ & $\begin{array}{l}\text { RS } 23 \\
\text { R } 22\end{array}$ & $98 \%$ & $400 \mathrm{mg}$ bid & Tomotherapy & $\begin{array}{l}50 \mathrm{~Gy} / 5 \mathrm{~F} \text { or } \\
10 \mathrm{~F}\end{array}$ & \\
\hline Wada [21] & $\begin{array}{l}\text { Inters Med, } \\
2018\end{array}$ & $\begin{array}{l}\text { Kyushu } \\
\text { Medical }\end{array}$ & 2009-2015 & Comparative & $\begin{array}{l}\text { Advanced } \\
\text { HCC (MVI, } \\
\text { EHM) }\end{array}$ & $\begin{array}{l}\text { RS } 15 \\
\text { S } 47\end{array}$ & $100 \%$ & $\begin{array}{l}\text { starting } 800 \\
\text { mg/day }\end{array}$ & 3DCRT & M50 (30-60) & \\
\hline Zhao [40] & $\begin{array}{c}\text { Frontier Oncol, } \\
2019\end{array}$ & $\begin{array}{l}\text { Peking } \\
\text { University, }\end{array}$ & 2015-2018 & Comparative & $\begin{array}{l}\text { HCC with } \\
\text { MVI }\end{array}$ & $\begin{array}{l}\text { TRS } 28 \\
\text { TR } 35\end{array}$ & $100 \%$ & $400 \mathrm{mg}$ bid & IMRT & $\begin{array}{c}5054 \mathrm{~Gy}, \\
\text { conventional } \\
\text { fraction }\end{array}$ & TACE \\
\hline Kang [35] & $\begin{array}{l}\text { Chin J Clin } \\
\text { Oncol, } 2013\end{array}$ & $\begin{array}{l}\text { Navy General } \\
\text { Hospital, } \\
\text { China }\end{array}$ & 2007-2009 & Comparative & $\begin{array}{l}\text { Recurrent and } \\
\text { metastatic } \\
\text { HCC }\end{array}$ & $\begin{array}{l}\text { RSh } 32 \\
\text { Rh } 39\end{array}$ & $64.8 \%$ & $400 \mathrm{mg}$ bid & $\underset{\text { SBRT }}{\text { Gammaknife }}$ & $\begin{array}{c}36-50 \text { Gy, } \\
10-13 \text { fractions }\end{array}$ & Hyperthermia \\
\hline Liu [38] & $\begin{array}{c}\text { ASTRO } \\
\text { abstract, } 2020\end{array}$ & $\begin{array}{c}\text { Chang Gung } \\
\text { Memorial } \\
\text { hospital, } \\
\text { Taiwan }\end{array}$ & 2007-2017 & Comparative & $\begin{array}{l}\text { Locally } \\
\text { advanced } \\
\text { HCC }\end{array}$ & $\begin{array}{c}\text { RS 73 } \\
\text { R 73, } \\
\text { propensity } \\
\text { matched }\end{array}$ & & & & & \\
\hline Zhang [39] & $\begin{array}{c}\text { World J } \\
\text { Gastroenterol, } \\
2017\end{array}$ & $\begin{array}{l}\text { Fudan Univ, } \\
\text { China }\end{array}$ & 2009-2015 & Comparative & HCC MPVTT & $\begin{array}{l}\text { TRS } 37 \\
\text { TS } 31\end{array}$ & $86.8 \%$ & $400 \mathrm{mg}$ bid & BrachyTx $\left(\mathrm{I}^{125}\right)$ & $\begin{array}{c}\text { mean } \\
\text { accumulated } \\
\text { dose } 62.9\end{array}$ & TACE \\
\hline Brade [16] & $\begin{array}{c}\text { Int J Radiat } \\
\text { Biol Phys, } 2016\end{array}$ & $\begin{array}{l}\text { Princess } \\
\text { Margaret } \\
\text { Hospital, } \\
\text { Canada }\end{array}$ & 2009-2012 & Single arm & $\begin{array}{c}\text { HCC not } \\
\text { amenable for } \\
\text { other local Tx } \\
\text { (PVT 63\%) }\end{array}$ & 15 & 100 & $\begin{array}{c}200 \mathrm{mg} \\
\text { OD-400 mg } \\
\text { BID }\end{array}$ & SBRT & $30-51 \mathrm{~Gy} / 6 \mathrm{~F}$ & \\
\hline Cha-2 [19] & & & & & EHM & 5 & & $\begin{array}{l}400 \mathrm{mg} \mathrm{bid} \\
(60 \%) 200 \mathrm{mg} \\
\text { bid }(40 \%)\end{array}$ & Tomotherapy & $\begin{array}{c}\text { M50.4 } \\
(30-58.42)\end{array}$ & \\
\hline Chen B [36] & $\begin{array}{c}\text { ASTRO } \\
\text { abstract, } 2019\end{array}$ & $\begin{array}{l}\text { Peking } \\
\text { University, }\end{array}$ & 2010-2016 & Single arm & $\begin{array}{l}\text { HCC with } \\
\text { MVI }\end{array}$ & 8 & 100 & & IMRT & M50 (28-66) & \\
\hline Chen WS [18] & $\begin{array}{c}\text { Int J Radiat } \\
\text { Biol Phys, } 2014\end{array}$ & $\begin{array}{l}3 \text { hospitals in } \\
\text { Taiwan }\end{array}$ & 2010-2013 & Single arm & $\begin{array}{l}\text { Locally } \\
\text { advanced } \\
\text { HCC }\end{array}$ & 40 & $100 \%$ & $\begin{array}{l}\text { starting } 400 \\
\text { mg bid }\end{array}$ & IMRT & $\begin{array}{l}50-60 \text { Gy in } \\
2-2.5 \text { Gy/F }\end{array}$ & \\
\hline $\mathrm{Li}$ [37] & $\begin{array}{l}\text { J Cancer Res } \\
\text { Clin Oncol, } \\
2010\end{array}$ & $\begin{array}{l}\text { Sun Yat-Sen } \\
\text { Univ, Taiwan }\end{array}$ & 2006-2009 & Single arm & $\begin{array}{l}\text { Lung } \\
\text { metastases }\end{array}$ & 8 & & $400 \mathrm{mg}$ bid & BrachyTx $\left(\mathrm{I}^{125}\right)$ & $\begin{array}{c}\text { Minimial } \\
\text { peripheral } \\
\text { dose 120-160 } \\
\text { Gy }\end{array}$ & \\
\hline
\end{tabular}

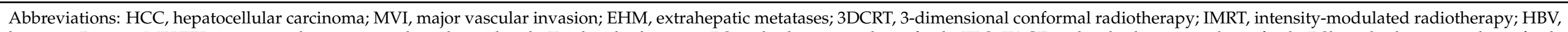

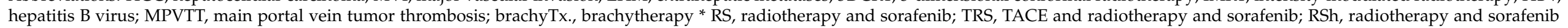
and hyperthermia. 
Table 2. Clinical results of studies included.

\begin{tabular}{|c|c|c|c|c|c|c|c|c|}
\hline Author & RT target & No. of Patients * & $\begin{array}{c}\text { Reliable } \\
\text { Comparability }\end{array}$ & OS & $\begin{array}{c}\text { Grade } \geq 3 \\
\text { Toxicity: GI }\end{array}$ & Hepatologic & Hematologic & Dermatologic \\
\hline Sun & EHM (lung mets) & $\begin{array}{l}\text { RS } 23 \\
\text { R } 22\end{array}$ & No & $\begin{array}{c}\text { RS: } 91.1 \%(1 \mathrm{y}) \\
78.8 \%(2 \mathrm{y}) \\
\text { R: } 66.8 \%(1 \mathrm{y}) \\
30.4 \%(2 \mathrm{y}) \\
(p=0.007)\end{array}$ & RS, R: $0 \%$ & RS, R: $0 \%$ & RS, R: $0 \%$ & RS, R: $0 \%$ \\
\hline Wada & $\begin{array}{l}\text { EHM or MVI (not } \\
\text { intrahepatic) }\end{array}$ & $\begin{array}{l}\text { RS } 15 \\
\text { S } 47\end{array}$ & Yes & $\begin{array}{c}\text { RS: M31.2mo., } \\
\text { 93.3\% (1 y), 56.9\% } \\
(2 \mathrm{y}) \\
\text { S: M12.1mo., } \\
47.9 \%(1 \mathrm{y}), \\
12.4 \%(2 \mathrm{y}) ; \\
(p<0.01)\end{array}$ & $\begin{array}{l}\text { RS: } 6.7 \% \\
\text { S: } 2.2 \% \\
(p=\mathrm{NS})\end{array}$ & $\begin{array}{c}\text { RS: } 0 \% \\
\text { S: } 6.4 \% \\
(p=0.23)\end{array}$ & $\begin{array}{l}\text { RS: } 0 \% \\
\text { S: } 0 \%\end{array}$ & $\begin{array}{c}\text { RS: } 0 \% \\
\text { S: } 4.3 \% \\
(p=0.24)\end{array}$ \\
\hline Zhao & Liver HCC & $\begin{array}{l}\text { TRS } 28 \\
\text { TR } 35\end{array}$ & Yes & $\begin{array}{c}\text { TRS: M19 mo } \\
\text { TR: M15.2 mo } \\
(p=0.094)\end{array}$ & $\begin{array}{l}\text { TRS: } 0 \% \\
\text { TR: } 0 \%\end{array}$ & $\begin{array}{c}\text { TRS: } 10.7 \% \\
\text { TR: } 11.4 \% \\
(p=1.0)\end{array}$ & $\begin{array}{c}\text { TRS: } 14.3 \% \\
\text { TR: } 17.1 \% \\
(p=1.0)\end{array}$ & $\begin{array}{l}\text { TRS: } 0 \% \\
\text { TR: } 0 \%\end{array}$ \\
\hline Kang & $\begin{array}{c}\text { EHM or Liver } \\
\text { HCC }\end{array}$ & $\begin{array}{l}\text { RSh } 32 \\
\text { Rh } 39\end{array}$ & Yes & $\begin{array}{c}\text { RSh: } 62.5 \%(1 \mathrm{y}) \\
\text { Rh: } 41.2 \%(1 \mathrm{y}) \\
(p=0.048)\end{array}$ & RSh, Rh: $0 \%$ & Not assessable & RSh, Rh: $0 \%$ & RSh, Rh: $0 \%$ \\
\hline Liu & Liver HCC & $\begin{array}{c}\text { RS } 73 \\
\text { R 73, propensity } \\
\text { matched }\end{array}$ & Yes & $\begin{array}{c}\text { RS: M9.6 } \\
\text { R: M9.9 } \\
(p=0.544)\end{array}$ & & & & \\
\hline Zhang & PVT only & $\begin{array}{l}\text { TRS } 37 \\
\text { TS } 31\end{array}$ & Yes & $\begin{array}{c}\text { TRS: } 54.3 \%(1 \mathrm{y}) \\
14.1 \%(2 \mathrm{y}) \\
\text { TS: } 0 \%(1 \mathrm{y}) \\
(p<0.001)\end{array}$ & $\begin{array}{c}\text { TRS: } 11.5 \% \\
\text { (diarrhea) } \\
\text { TS: } 3.6 \% \text { (diarrhea) } \\
(p=\text { NS) }\end{array}$ & $\begin{array}{l}\text { Grade not } \\
\text { assessed }\end{array}$ & & $\begin{array}{c}\text { TRS: } 7.1 \% \text { (HFS) } \\
\text { TS: } 3.6 \% \text { (HFS) } \\
\quad(p=\text { NS) }\end{array}$ \\
\hline Brade & Liver HCC & 15 & NA & $\begin{array}{c}\text { M26.3 mo, } 62.5 \% \\
(1 \mathrm{y})\end{array}$ & $\begin{array}{c}20 \% \text { (GI bleeding } \\
\text { and SBO) }\end{array}$ & $\begin{array}{l}20 \% \text { (LFT } \\
\text { elevation) }\end{array}$ & $\begin{array}{l}33.3 \% \text { (thrombocy- } \\
\text { topenia) }\end{array}$ & $0 \%$ \\
\hline $\begin{array}{l}\text { Cha } \\
\text { Cha-2 }\end{array}$ & $\begin{array}{l}\text { Liver HCC } \\
\text { EHM }\end{array}$ & $\begin{array}{c}13 \\
5\end{array}$ & $\begin{array}{l}\text { NA } \\
\text { NA }\end{array}$ & $\begin{array}{l}\text { M7.8 mo, 35\% (1 y) } \\
\text { M15.7 mo, 60\% } \\
(1 \mathrm{y})\end{array}$ & $5.6 \%$ (DU bleed) & $\begin{array}{l}5.6 \% \text { (LFT } \\
\text { elevation) }\end{array}$ & $\begin{array}{c}16.7 \% \text { (thrombocy- } \\
\text { topenia) }\end{array}$ & $16.7 \%$ (HFS) \\
\hline Chen B & Liver HCC & 8 & NA & & & & & \\
\hline Chen WS & Liver HCC & 40 & NA & $\begin{array}{c}\mathrm{M} 14 \mathrm{mo}, \\
52.5 \%(1 \mathrm{y}), 32 \% \\
(2 \mathrm{y})\end{array}$ & $5 \%$ (diarrhea) & $22.50 \%$ & $0 \%$ & 7.5\% (HFS) \\
\hline $\mathrm{Li}$ & EHM (lung mets) & 8 & NA & $\begin{array}{c}\text { M21 mo, } \\
100 \%(1 \mathrm{y}), 50 \% \\
(2 \mathrm{y})\end{array}$ & 0 & 0 & 0 & 0 \\
\hline
\end{tabular}

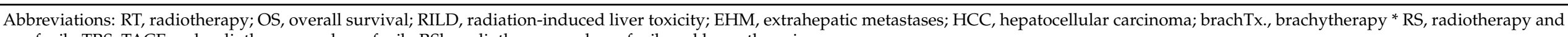
sorafenib; TRS, TACE and radiotherapy and sorafenib; RSh, radiotherapy and sorafenib and hyperthermia. 


\subsection{Synthesized Results and Qualitative Analyses of Endpoints}

Pooled analyses of grade $\geq 3$ toxicities were performed categorically: gastrointestinal (e.g., duodenal or gastric ulcer and/or perforation, abdominal pain, severe nausea and/or vomiting), hepatologic (e.g., elevation of liver function, symptoms of liver decompensation), hematologic (e.g., thrombocytopenia, leukopenia, anemia), and dermatologic complications were measured. Pooled rates of gastrointestinal, hepatologic, hematologic, and dermatologic grade $\geq 3$ toxicities were $8.1 \%$ (95\% confidence interval (CI): 4.8-13.5; heterogeneity: $p=0.444, \mathrm{I}^{2}=\sim 0 \%$ ), $12.9 \%$ (95\% CI: 7.1-22.1, $\left.p=0.259, \mathrm{I}^{2}=22.4 \%\right), 9.1 \%$ (95\% CI: $\left.3.8-20.3, p=0.045, \mathrm{I}^{2}=51.3 \%\right)$, and $6.8 \%\left(95 \%\right.$ CI: 3.8-11.7, $\left.p=0.619, \mathrm{I}^{2}=\sim 0 \%\right)$, respectively.

In subgroup analyses, pooled grade $\geq 3$ hepatologic toxicity rates were significantly lower in studies targeting non-intrahepatic than intrahepatic lesions (3.3\% vs. $17.1 \%$, $p=0.041)$, as targets of EBRT, and pooled hematologic toxicity rates showed a similar trend $(3.3 \%$ vs. $16.0 \%, p=0.078)$. Pooled rates of gastrointestinal and dermatologic grade $\geq 3$ complications were not significantly different between the subgroups. Pooled analyses regarding OS were performed in six comparative studies, and concurrent treatment was more beneficial than non-concurrent treatment (odds ratio: 3.3, 95\% CI: $1.3-8.59$, p for odds ratio: 0.015 ; heterogeneity $p=0.002, \mathrm{I}^{2}=73.5$ ).

The heterogeneity of pooled analyses was non-significant except for the pooled analyses of OS and hematologic toxicities in all studies and studies targeting intrahepatic lesions. Publication bias assessment was not performed, as all analyses included fewer than 10 studies. The pooled results are shown in Table 3 and are depicted in Figure 2 as forest plots.

Table 3. Pooled rates of grade 3 or higher toxicities.

\begin{tabular}{|c|c|c|c|c|c|c|}
\hline $\begin{array}{l}\text { Subject } \\
\text { Studies }\end{array}$ & $\begin{array}{l}\text { No. of } \\
\text { Studies }\end{array}$ & $\begin{array}{c}\text { Patients Underwent } \\
\text { Concurrent } \\
\text { Treatment }\end{array}$ & Heterogeneity $p$ & $\mathrm{I}^{2}$ & $\begin{array}{l}\text { Pooled Rate } \\
\text { (95\% CI) }\end{array}$ & $\begin{array}{c}\text { Subgroup } \\
\text { Comparison } p\end{array}$ \\
\hline \multicolumn{7}{|l|}{$\begin{array}{l}\text { Gastrointestinal } \\
\text { toxicity }\end{array}$} \\
\hline All studies & 9 & 217 & 0.444 & $\sim 0 \%$ & $8.1 \%(4.8-13.5)$ & NA \\
\hline $\begin{array}{c}\text { Non- } \\
\text { intrahepatic }\end{array}$ & 4 & 83 & 0.649 & $\sim 0 \%$ & $8.6 \%(4.0-17.7)$ & 0.859 \\
\hline \multicolumn{7}{|l|}{$\begin{array}{l}\text { Hepatologic } \\
\text { toxicity }\end{array}$} \\
\hline All studies & 7 & 148 & 0.259 & $22.4 \%$ & $12.9 \%(7.1-22.1)$ & NA \\
\hline $\begin{array}{c}\text { Non- } \\
\text { intrahepatic }\end{array}$ & 3 & 46 & 0.882 & $\sim 0 \%$ & $3.3 \%(0.7-14.8)$ & 0.041 \\
\hline \multicolumn{7}{|l|}{$\begin{array}{l}\text { Hematologic } \\
\text { toxicity }\end{array}$} \\
\hline All studies & 8 & 180 & 0.045 & $51.3 \%$ & $9.1 \%(3.8-20.3)$ & NA \\
\hline $\begin{array}{c}\text { Non- } \\
\text { intrahepatic }\end{array}$ & 3 & 46 & 0.882 & $\sim 0 \%$ & $3.3 \%(0.7-14.8)$ & 0.078 \\
\hline \multicolumn{7}{|l|}{$\begin{array}{l}\text { Dermatologic } \\
\text { toxicity }\end{array}$} \\
\hline All studies & 9 & 217 & 0.619 & $\sim 0 \%$ & $6.8 \%(3.8-11.7)$ & \multirow{4}{*}{0.485} \\
\hline $\begin{array}{c}\text { Non- } \\
\text { intrahepatic }\end{array}$ & 4 & 83 & 0.839 & $\sim 0 \%$ & $5.4 \%(2.1-13.3)$ & \\
\hline Intrahepatic & 4 & 102 & 0.321 & $14.3 \%$ & 8.3\% (3.6-18.1) & \\
\hline Overall survival & 6 & 455 & 0.002 & $73.5 \%$ & $\begin{array}{c}\text { OR: } 3.3(1.3-8.59 \\
p=0.015)\end{array}$ & \\
\hline
\end{tabular}


(A)

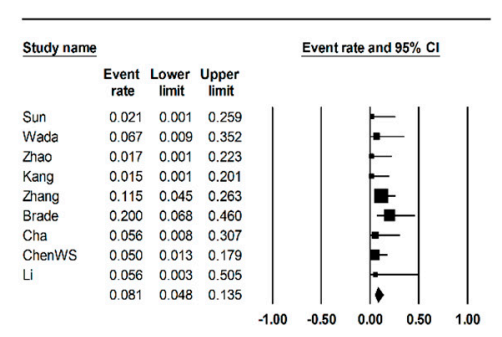

(C)

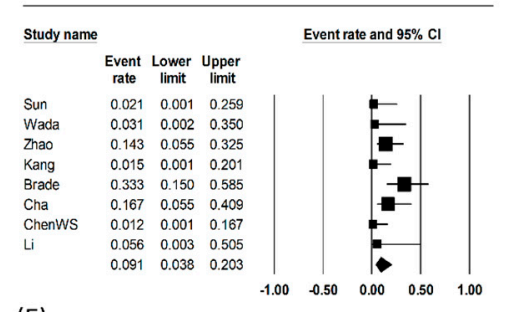

(E)

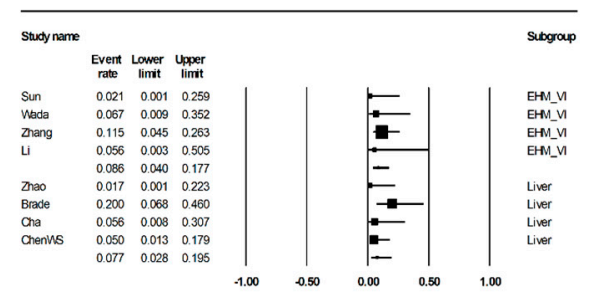

(G)

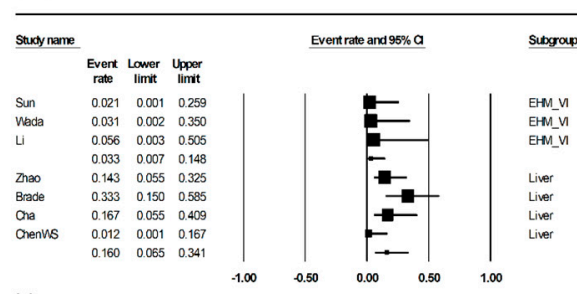

(1)

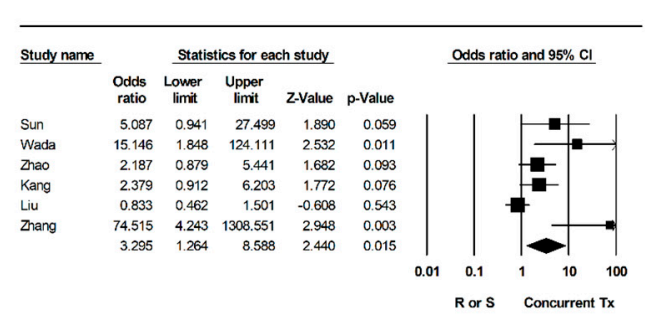

(B)

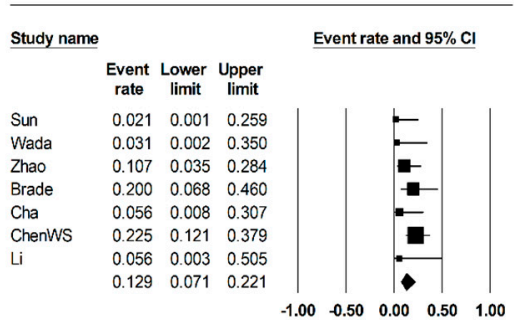

(D)

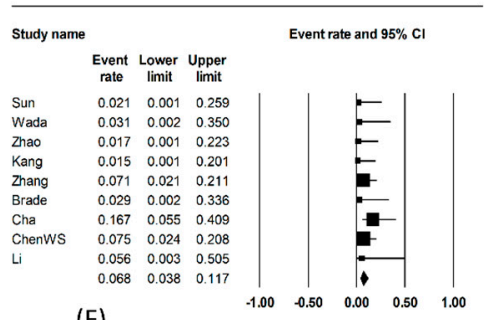

(F)

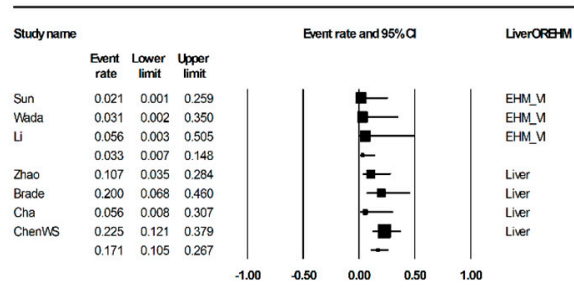

(H)

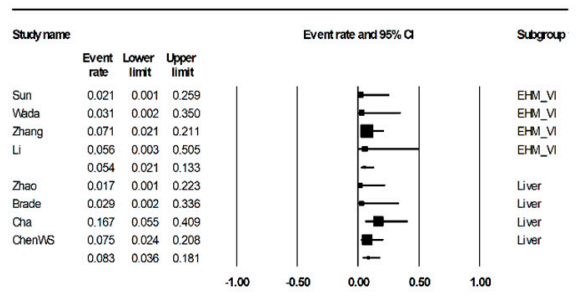

Figure 2. Forest plots of pooled analyses of all included studies regarding (A) gastrointestinal, (B) hepatologic, (C) hematologic, (D) dermatologic grade $\geq 3$ toxicities; subgroup analyses comparing studies with RT targets of intrahepatic and non-intrahepatic lesions, regarding (E) gastrointestinal, (F) hepatologic, $(\mathbf{G})$ hematologic, $(\mathbf{H})$ dermatologic grade $\geq 3$ toxicities. (I) Overall survival comparing concurrent and non-concurrent arms.

\subsection{Qualitative Assessment of Grade 4 or 5 and Descriptive Toxicities}

Among nine studies with available data, four studies $[16,18,19,39]$ reported few cases of grade 4 or 5 toxicities, whereas other studies did not. Brade et al. [16] reported lethal toxicity caused by upper gastrointestinal bleeding and tumor rupture. Among six comparative studies, two studies reported higher incidence of grade 1 or 2 hematologic or dermatologic toxicities in combination arms [21,40]. Kang et al. [35]. reported that grade 1 or 2 hematologic toxicity, fatigue and nausea are not different between comparative arms. Liu et al. [38]. also reported that toxicity was not different regarding radiation-induced 
liver toxicity and gastrointestinal bleeding. On the other hand, Zhang et al. [39] reported that additional brachytherapy showed benefits in terms of symptoms related to portal hypertension. The qualitative interpretation of toxicities is shown in detail in Table 4.

Table 4. Qualitative interpretation of toxicities.

\begin{tabular}{|c|c|c|c|c|}
\hline Author & RT Target & No. of Patients * & Grade 4 or 5 Toxicities RILD & Qualitative Interpretation \\
\hline Sun & EHM (lung mets) & $\begin{array}{l}\text { RS } 23 \\
\text { R } 22\end{array}$ & $\begin{array}{c}0 \% \\
\text { no RILD }\end{array}$ & $\begin{array}{c}\text { All toxicities were G1 or } \\
2 \text { toxicities }\end{array}$ \\
\hline Wada & $\begin{array}{l}\text { EHM or MVI (not } \\
\text { intrahepatic) }\end{array}$ & $\begin{array}{l}\text { RS } 15 \\
\text { S } 47\end{array}$ & & $\begin{array}{c}\text { Grade } 1 \text { or } 2 \text { hematologic, } \\
\text { dermatologic adverse events } \\
\text { were higher in the RS group } \\
\text { Overall grade } \geq 3 \text { toxicity } \\
\text { incidences are similar ( } 20 \% \text { vs. } \\
19.2 \%, p=\mathrm{NS})\end{array}$ \\
\hline Zhao & Liver HCC & $\begin{array}{l}\text { TRS } 28 \\
\text { TR } 35\end{array}$ & $\begin{array}{c}0 \% \\
\text { no RILD }\end{array}$ & $\begin{array}{l}\text { All skin reactions and HFS } \\
\text { were G1 or } 2 \text { toxicities, but } \\
\text { these toxicities were of a } \\
\text { higher grade with TRS }(92.9 \% \\
\text { \& } 17.9 \% \text { vs. } 68.6 \% \text { \& } 0 \%)\end{array}$ \\
\hline Kang & EHM or Liver HCC & $\begin{array}{l}\text { RSh } 32 \\
\text { Rh } 39\end{array}$ & $\begin{array}{c}0 \% \\
\text { RILD not assessed }\end{array}$ & $\begin{array}{c}\text { No significant difference in G1 } \\
\text { or } 2 \text { BM suppression, fatigue, } \\
\text { nausea between arms } \\
\text { Overall G3 complication } 9.4 \%\end{array}$ \\
\hline Liu & Liver HCC & $\begin{array}{l}\text { RS } 73 \\
\text { R 73, propensity } \\
\text { matched }\end{array}$ & & $\begin{array}{l}\text { No significant difference in } \\
\text { RILD and GI bleeding }\end{array}$ \\
\hline Zhang & PVT only & $\begin{array}{l}\text { TRS } 37 \\
\text { TS } 31\end{array}$ & $\begin{array}{c}1 \text { case of HTN G4 }(3.2 \%) \\
\text { in TRS } \\
\text { no lethal toxicity }\end{array}$ & $\begin{array}{c}\text { Adding brachyTx. improved } \\
\text { portal hypertension symptoms } \\
\text { (new ascites, liver dysfunction), } \\
\text { and OS ( } p<0.001,1 \text { yr OS } \\
54.3 \% \text { vs. } 0 \% \text { ) }\end{array}$ \\
\hline Brade & Liver HCC & 15 & $\begin{array}{c}1 \text { case of liver enzyme } \\
\text { change G4; } \\
1 \text { case of SBO G4 } \\
1 \text { case of upper GI bleeding, } \\
\text { rupture and death }(6.7 \%)\end{array}$ & \\
\hline Cha & Liver or EHM & 18 & $\begin{array}{c}1 \text { case of G4 thrombocytopenia } \\
(5.6 \%) \\
\text { no RILD }\end{array}$ & \\
\hline Chen B & Liver HCC & 8 & $\begin{array}{c}\text { No lethal toxicity } \\
\text { no RILD }\end{array}$ & \\
\hline Chen WS & Liver HCC & 40 & $\begin{array}{l}4 \text { cases }(11.1 \%) \text { G } 4-5 \text { hepatic } \\
\text { toxicity } \\
\text { RILD } 15 \% \text { ( } 6 \text { cases, } 3 \text { of which } \\
\text { died without tumor } \\
\text { progression) }\end{array}$ & \\
\hline $\mathrm{Li}$ & EHM (lung mets) & 8 & $\begin{array}{l}\text { No RILD } \\
\text { no lethal toxicity }\end{array}$ & \\
\hline
\end{tabular}

Abbreviations: RT, radiotherapy; RILD, radiation-induced liver disease; EHM, extrahepatic metastases; MVI, major vessel invasion; HCC, hepatocellular carcinoma; BM, bone marrows; HFS, hand-foot syndrome; SBO, small bowel obstruction; GI, gastrointestinal * RS, radiotherapy and sorafenib; TRS, TACE and radiotherapy and sorafenib; RSh, radiotherapy and sorafenib and hyperthermia.

\section{Discussion}

Our findings suggest that concurrent application of sorafenib and EBRT is a feasible option for treating advanced HCC. Several types of adverse events of grade $\geq 3$ occurred in $6 \%-13 \%$ of cases. Regarding the pooled analysis of OS, the combination therapy showed significantly greater benefit than did the non-concurrent therapy. On the basis of the above results, this study suggests that the application of concurrent therapy should be considered for advanced stages, in which available treatment options are limited. 
The magnitude of toxicities and correlating recommendations for concurrent treatment vary among researchers. Brade et al. [16] from the Princess Margarette Hospital reported that the rate of grade $\geq 3$ hepatotoxicity and gastrointestinal toxicity was approximately $20 \%$, and hematotoxicity was as high as $33.3 \%$. They did not recommend concurrent therapy because of the high risks of toxicities, assuming that toxicity was higher than that in their prior SBRT study with a similar design regarding RT modality. Chen et al. [18] reported a $22.5 \%$ hepatotoxicity of grade $\geq 3$ and did not recommend the application of combination therapy. These studies have been published in reputed journals in the field of radiation oncology and have influenced many clinical decisions and research study designs.

In contrast, other studies included in the present meta-analysis were more favorable for the application of combination therapy. In terms of toxicities, comparative studies did not report excessive serious toxicities related to the concurrent application [20,21,35,38-40], and single-arm studies reported that the degree of complication was acceptable and severe toxicity (grade $\geq 4$ ) was rare [19,36,37]. Furthermore, Zhang et al. [39] reported that the concurrent application of brachytherapy to the portal vein significantly reduced symptoms due to liver compensation. Five of six comparative studies $[20,21,35,39,40]$ reported that combination therapy could increase OS. Four of these five studies $[21,35,39,40]$ had reliable comparability, which means that the clinical characteristics were comparable between groups, or the concurrent treatment arm did not have relatively favorable clinical indicators. Theoretically, spatial cooperation in which EBRT targets macroscopic disease and sorafenib controls latent microscopic disease can be the basis for combination therapy [8]. Furthermore, sorafenib inhibits DNA damage repair in cancer cells in the tumor microenvironment and enhances the oxygen effect through normalization of the surviving tumor vasculature $[14,15]$. Sorafenib targets multiple targets (Vascular endothelial growth factor, Platelet-derived growth factor receptor, and Raf), reducing tumor-stromal interactions related to carcinogenesis and metastatic capacity, and affects metabolic capacity related to tumorigenicity [41,42], whereas EBRT induces mitotic death via DNA damage as well as immunogenic stimulation [43]. From a practical perspective, delaying the application of systemic agents during the 1-2-month period required for the planning and conducting of EBRT might lead to the development of latent microscopic disease while treating advanced HCCs. Therefore, the concurrent use of sorafenib and EBRT might be justified based on the above clinical results and preclinical rationale. Our results might also support the conduction of clinical trials, for instance, in which concurrent treatment is applied to patients with predictive factors related to poor outcomes after sorafenib [44].

Notably, subgroup analyses showed that rates of grade $\geq 3$ hepatotoxicities and gastrointestinal toxicities were significantly different; these were lower in study groups targeting non-intrahepatic lesions than intrahepatic lesions. Currently, it might be difficult to recommend the application of concurrent treatment for all advanced HCCs because the literature regarding the feasibility and efficacy of concurrent treatment is insufficient, and some studies have reported risks of possible excessive toxicities $[16,18]$. However, considering the results of the present meta-analysis and the preclinical rationale mentioned above, we recommend the application of combination therapy to treat metastatic lesions or vascular tumor involvement as a target of EBRT. Combination therapy in such a disease situation is less likely to cause excessive toxicities, especially hepatotoxicity, which is a major concern. Notably, two studies reported a significant risk of hepatotoxicity, and the median value of the largest tumor size was $8.2-8.7 \mathrm{~cm}[16,18]$. Targeting such large intrahepatic tumors inevitably leaves less normal liver volume unirradiated, which might result in excessive hepatotoxicity. Thus, concurrent treatment should be administered cautiously, considering tumor size and liver function reserve. In addition, skin reactions should be cautiously monitored when concurrent treatment is applied because excessive dermatologic toxicity has been reported in other case series [17], and, among the included studies, two of them [21,40] reported that grade 1 or 2 skin reactions were increased in addition to concurrent modality. Future studies are warranted to identify the mechanism of possible excessive toxicities caused by the concurrent application of sorafenib and EBRT. 
The limitations of this study are as follows. Meta-analysis of observational studies is controversial because differences in study design and clinical characteristics might affect pooled estimates [45]. However, in the field of oncology, not all clinical decisions can be made on the basis of data from randomized studies. For intractable disease situations where standard treatment is not established and related literature is scarce, a meta-analysis of observational studies could be one of the few available options to suggest therapeutic decisions [46,47]. Efforts to improve the quality of meta-analysis, such as heterogeneity analysis, formal quality assessment, and sensitivity analyses, are recommended [46,48]. To optimize the present meta-analysis, we conducted statistical complements, as well as qualitative assessments. The heterogeneity in the pooled analyses was mostly low, suggesting that these results were reliable for clinical decisions. The inclusion of a small number of studies is also a limitation. We hope that this study would encourage further research to accumulate clinical evidence for navigating optimal indications of concurrent EBRT and sorafenib.

\section{Conclusions}

The present meta-analysis of the literature generates the clinical hypothesis that concurrent treatment of advanced HCC with sorafenib and EBRT could be a viable option. Such a modality can be applied to target metastatic lesions or vessel tumor involvement with RT. Treatment of intrahepatic lesions with a combined treatment modality should be performed cautiously by considering the target size and hepatic function reserve. Considering that concurrent treatment can increase treatment efficiency through spatial cooperation or radiosensitization and help avoid delays in systemic treatment, the clinical application should be more openly considered, and more future studies should be conducted.

Supplementary Materials: The following are available online at https:/ /www.mdpi.com/article/10 .3390/ cancers13122912/s1, Table S1: Scoring sheet according to New-Castle Ottawa scale. Supplementary Document 1: Draft of search strategy.

Author Contributions: C.H.R. conceptualized the study, wrote and edited the original draft, and performed data curation; S.P. performed data curation and recruitment; I.-S.S. performed the statistical analysis as a biostatistician; and W.S.Y. supervised the study. All authors have read and agreed to the published version of the manuscript.

Funding: This study was supported by the National Research Fund of Korea (NRF-2019M2D2A1A01 031560). The funders had no role in the study design, data collection and analysis, decision to publish, or preparation of the manuscript.

Institutional Review Board Statement: Not applicable.

Informed Consent Statement: Not applicable.

Data Availability Statement: The authors confirm that the data supporting the findings of this study are available within the article and/or its supplementary materials.

Acknowledgments: We appreciate the dedicated contribution of Hu Jing Hua, who majored in Korean Language Translation at Chonnam National University, Jeollanamdo, Korea, for dedicated Chinese language translation.

Conflicts of Interest: The authors declare no conflict of interest.

Ethical Consideration and Consent for Publication: Ethical approval was not required because this study retrieved and synthesized data that have already been published. 


\section{References}

1. Cassim, S.; Raymond, V.A.; Lacoste, B.; Lapierre, P.; Bilodeau, M. Metabolite profiling identifies a signature of tumorigenicity in hepatocellular carcinoma. Oncotarget 2018, 9, 26868-26883. [CrossRef]

2. Dai, J.; Zhao, J.; Du, Y.; Zhuang, L.; McNeil, E.B.; Chongsuvivatwong, V. Adherence to Hepatocellular Carcinoma Surveillance and Perceived Barriers Among High-Risk Chronic Liver Disease Patients in Yunnan, China. Cancer Manag. Res. 2020, 12, 6209-6220. [CrossRef]

3. Ayuso, C.; Rimola, J.; García-Criado, A. Imaging of HCC. Abdom. Imaging 2012, 37, 215-230. [CrossRef]

4. Burrel, M.; Llovet, J.M.; Ayuso, C.; Iglesias, C.; Sala, M.; Miquel, R.; Caralt, T.; Ayuso, J.R.; Solé, M.; Sanchez, M.; et al. MRI angiography is superior to helical CT for detection of HCC prior to liver transplantation: An explant correlation. Hepatology 2003, 38, 1034-1042. [CrossRef] [PubMed]

5. Chalasani, N.; Horlander, J.C., Sr.; Said, A.; Hoen, H.; Kopecky, K.K.; Stockberger, S.M., Jr.; Manam, R.; Kwo, P.Y.; Lumeng, L. Screening for hepatocellular carcinoma in patients with advanced cirrhosis. Am. J. Gastroenterol. 1999, 94, 2988-2993. [CrossRef] [PubMed]

6. Wu, Q.; Qin, S.K. Features and treatment options of Chinese hepatocellular carcinoma. Chin. Clin. Oncol. 2013, 2, 38. [PubMed]

7. Lee, J.; Yoon, W.S.; Koom, W.S.; Rim, C.H. Role of local treatment including radiotherapy in Barcelona Clinic of Liver Cancer stage C patients: A nationwide cohort analysis in South Korea. Cancer Manag. Res. 2019, 11, 1373-1382. [CrossRef] [PubMed]

8. Lawrence, T.S.; Blackstock, A.W.; McGinn, C. (Eds.) The Mechanism of Action of Radiosensitization of Conventional Chemotherapeutic Agents; Seminars in radiation oncology; Elsevier: Amsterdam, The Netherlands, 2003.

9. Wilson, G.D.; Bentzen, S.M.; Harari, P.M. (Eds.) Biologic Basis for Combining Drugs with Radiation; Seminars in radiation oncology; Elsevier: Amsterdam, The Netherlands, 2006.

10. Llovet, J.M.; Ricci, S.; Mazzaferro, V.; Hilgard, P.; Gane, E.; Blanc, J.F.; De Oliveira, A.C.; Santoro, A.; Raoul, J.L.; Forner, A.; et al. Sorafenib in advanced hepatocellular carcinoma. N. Engl. J. Med. 2008, 359, 378-390. [CrossRef] [PubMed]

11. Lee, H.A.; Park, S.; Seo, Y.S.; Yoon, W.S.; Rim, C.H.; Group KLCS. Benefits of Local Treatment Including External Radiotherapy for Hepatocellular Carcinoma with Portal Invasion. Biology 2021, 10, 326. [CrossRef] [PubMed]

12. Rim, C.H.; Kim, C.Y.; Yang, D.S.; Yoon, W.S. Comparison of radiation therapy modalities for hepatocellular carcinoma with portal vein thrombosis: A meta-analysis and systematic review. Radiother. Oncol. 2018, 129, 112-122. [CrossRef] [PubMed]

13. Rim, C.H.; Yoon, W.S. Leaflet manual of external beam radiation therapy for hepatocellular carcinoma: A review of the indications, evidences, and clinical trials. OncoTargets Ther. 2018, 11, 2865. [CrossRef]

14. Girard, N.; Mornex, F. Sorafenib and radiotherapy association for hepatocellular carcinoma. Cancer Radiother 2011, 15, 77-80. [CrossRef]

15. Yu, W.; Gu, K.; Yu, Z.; Yuan, D.; He, M.; Ma, N.; Lai, S.; Zhao, J.; Ren, Z.; Zhang, X.; et al. Sorafenib potentiates irradiation effect in hepatocellular carcinoma in vitro and in vivo. Cancer Lett. 2013, 329, 109-117. [CrossRef]

16. Brade, A.M.; Ng, S.; Brierley, J.; Kim, J.; Dinniwell, R.; Ringash, J.; Wong, R.R.; Cho, C.; Knox, J.; Dawson, L.A. Phase 1 trial of sorafenib and stereotactic body radiation therapy for hepatocellular carcinoma. Int. J. Radiat. Oncol. Biol. Phys. 2016, 94, 580-587. [CrossRef]

17. Chen, J.C.-H.; Liu, S.-H.; Yen, T.-Y.; Dai, K.-Y.; Hwang, J.-J.; Chen, Y.-J. Sorafenib-Induced Radiation in-Field Skin (SIRIS) Reaction: Unexpected Complication in Elder Hepatocellular Carcinoma Patients Received Sorafenib and Concurrent Radiation Therapy. Int. J. Gerontol. 2019, 13, 90-93. [CrossRef]

18. Chen, S.-W.; Lin, L.-C.; Kuo, Y.-C.; Liang, J.-A.; Kuo, C.-C.; Chiou, J.-F. Phase 2 study of combined sorafenib and radiation therapy in patients with advanced hepatocellular carcinoma. Int. J. Radiat. Oncol. Biol. Phys. 2014, 88, 1041-1047. [CrossRef]

19. Cha, J.; Seong, J.; Lee, I.J.; Kim, J.W.; Han, K.-H. Feasibility of sorafenib combined with local radiotherapy in advanced hepatocellular carcinoma. Yonsei Med. J. 2013, 54, 1178. [CrossRef] [PubMed]

20. Sun, T.; He, J.; Zhang, S.; Sun, J.; Zeng, M.; Zeng, Z. Simultaneous multitarget radiotherapy using helical tomotherapy and its combination with sorafenib for pulmonary metastases from hepatocellular carcinoma. Oncotarget 2016, 7, 48586. [CrossRef] [PubMed]

21. Wada, Y.; Takami, Y.; Matsushima, H.; Tateishi, M.; Ryu, T.; Yoshitomi, M.; Matsumura, T.; Saitsu, H. The safety and efficacy of combination therapy of sorafenib and radiotherapy for advanced hepatocellular carcinoma: A retrospective study. Intern. Med. 2018, 1345-1353. [CrossRef] [PubMed]

22. Lee, J.; Kim, W.C.; Yoon, W.S.; Rim, C.H. Implications of Radiotherapy Utilization in South Korea from 2010 to 2019. J. Korean Med. Sci. 2021, 36, e117. [CrossRef]

23. Rim, C.H.; Kim, H.J.; Seong, J. Clinical feasibility and efficacy of stereotactic body radiotherapy for hepatocellular carcinoma: A systematic review and meta-analysis of observational studies. Radiother. Oncol. 2019, 131, 135-144. [CrossRef] [PubMed]

24. Moher, D.; Altman, D.G.; Liberati, A.; Tetzlaff, J. PRISMA statement. Epidemiology 2011, 22, 128. [CrossRef]

25. Higgins, J.P.; Thomas, J.; Chandler, J.; Cumpston, M.; Li, T.; Page, M.J.; Welch, V.A. (Eds.) Cochrane Handbook for Systematic Reviews of Interventions; John Wiley \& Sons: Hoboken, NJ, USA, 2019.

26. Lefebvre, C.; Glanville, J.; Briscoe, S.; Littlewood, A.; Marshall, C.; Metzendorf, M.-I.; Noel-Storr, A.; Rader, T.; Shokraneh, F.; Thomas, J.; et al. Chapter 4: Searching for and selecting studies. In Cochrane Handbook for Systematic Reviews of Interventions Version 6.2; (updated February 2021); Higgins, J.P.T., Thomas, J., Chandler, J., Cumpston, M., Li, T., Page, M.J., Welch, V.A., Eds.; Cochrane: London, UK, 2021; Available online: www.training.cochrane.org/handbook (accessed on 2 February 2021). 
27. Reeves, B.C.; Deeks, J.J.; Higgins, J.P.T.; Shea, B.; Tugwell, P.; Wells, G.A. Chapter 24: Including non-randomized studies on intervention effects. In Cochrane Handbook for Systematic Reviews of Interventions Version 6.2; (updated February 2021); Higgins, J.P.T., Thomas, J., Chandler, J., Cumpston, M., Li, T., Page, M.J., Welch, V.A., Eds.; Cochrane: London, UK, 2021; Available online: www.training.cochrane.org/handbook (accessed on 2 February 2021).

28. Peterson, J.; Welch, V.; Losos, M.; Tugwell, P. The Newcastle-Ottawa Scale (NOS) for Assessing the Quality of Nonrandomised Studies in Meta-Analyses; University of Liverpool: Liverpool, UK, 2011.

29. Hozo, S.P.; Djulbegovic, B.; Hozo, I. Estimating the mean and variance from the median, range, and the size of a sample. BMC Med. Res. Methodol. 2005, 5, 1-10. [CrossRef] [PubMed]

30. Reeves, B.C.; Deeks, J.J.; Higgins, J.P.; Shea, B.; Tugwell, P.; Wells, G.A.; On Behalf of the Cochrane Non-Randomized Studies of Interventions Methods Group. Including non-randomized studies on intervention effects. In Cochrane Handbook for Systematic Reviews of Interventions; John Wiley \& Sons: Hoboken, NJ, USA, 2019; pp. 595-620.

31. Cochran, W.G. The Combination of Estimates from Different Experiments. Biometrics 1954, 10, 101-129. [CrossRef]

32. Higgins, J.P.T.; Thompson, S.G. Quantifying heterogeneity in a meta-analysis. Stat. Med. 2002, 21, 1539-1558. [CrossRef] [PubMed]

33. Egger, M.; Smith, G.D.; Schneider, M.; Minder, C. Bias in meta-analysis detected by a simple, graphical test. Br. Med. J. 1997, 315, 629-634. [CrossRef] [PubMed]

34. Richardson, M.; Garner, P.; Donegan, S. Interpretation of subgroup analyses in systematic reviews: A tutorial. Clin. Epidemiol. Glob. Health 2019, 7, 192-198. [CrossRef]

35. Kang, J.; Zhao, X.; Nie, Q.; Zhang, L.; Zhu, Q.; Li, Q.; Wang, R. Efficacy of Stereotactic body radiation therapy with gamma-knife combined with hyperthermia and targeted drug sorafenib for the treatment of recurrent and metastatic hepatocellular carcinoma. Chin. J. Clin. Oncol. 2013, 40, 1236-1239.

36. Chen, B.; Li, Y.; Wang, W.; Tan, Y.; Wang, S.; Zheng, X.; Chen, S.; Zhao, Y.; Jin, J.; Fang, H.; et al. Efficacy and Prognosis of Radiotherapy for Hepatocellular Carcinoma with Tumor Thrombosis in Main Portal Vein or/and Vena Cava. Int. J. Radiat. Oncol. Biol. Phys. 2019, 105, E215-E216. [CrossRef]

37. Li, C.; Zhang, F.; Zhang, W.; Zhang, L.; Huang, Z.; Wu, P. Feasibility of 125 I brachytherapy combined with sorafenib treatment in patients with multiple lung metastases after liver transplantation for hepatocellular carcinoma. J. Cancer Res. Clin. Oncol. 2010, 136, 1633-1640. [CrossRef]

38. Liu, C. Concurrent Sorafenib and Radiotherapy versus Radiotherapy Alone for Locally Advanced Hepatocellular Carcinoma. Int. J. Radiat. Oncol. Biol. Phys. 2020, 108, e580. [CrossRef]

39. Zhang, Z.-H.; Liu, Q.-X.; Zhang, W.; Ma, J.-Q.; Wang, J.-H.; Luo, J.-J.; Liu, L.-X.; Yan, Z.-P. Combined endovascular brachytherapy, sorafenib, and transarterial chemobolization therapy for hepatocellular carcinoma patients with portal vein tumor thrombus. World J. Gastroenterol. 2017, 23, 7735. [CrossRef] [PubMed]

40. Zhao, Y.; Zhu, X.; Wang, H.; Dong, D.; Gao, S.; Zhu, X.; Wang, W. Safety and efficacy of transcatheter arterial chemoembolization plus radiotherapy combined with sorafenib in hepatocellular carcinoma showing macrovascular invasion. Front. Oncol. 2019, 9, 1065. [CrossRef]

41. Cassim, S.; Raymond, V.A.; Dehbidi-Assadzadeh, L.; Lapierre, P.; Bilodeau, M. Metabolic reprogramming enables hepatocarcinoma cells to efficiently adapt and survive to a nutrient-restricted microenvironment. Cell Cycle 2018, 17, 903-916. [CrossRef]

42. Yang, J.D.; Nakamura, I.; Roberts, L.R. The tumor microenvironment in hepatocellular carcinoma: Current status and therapeutic targets. Semin. Cancer Biol. 2011, 21, 35-43. [CrossRef]

43. Lee, Y.H.; Tai, D.; Yip, C.; Choo, S.P.; Chew, V. Combinational Immunotherapy for Hepatocellular Carcinoma: Radiotherapy, Immune Checkpoint Blockade and Beyond. Front. Immunol. 2020, 11, 568759. [CrossRef]

44. Brunetti, O.; Gnoni, A.; Licchetta, A.; Longo, V.; Calabrese, A.; Argentiero, A.; Delcuratolo, S.; Solimando, A.G.; Casadei-Gardini, A.; Silvestris, N. Predictive and Prognostic Factors in HCC Patients Treated with Sorafenib. Medicina 2019, 55, 707. [CrossRef]

45. Stroup, D.F.; Berlin, J.A.; Morton, S.C.; Olkin, I.; Williamson, G.D.; Rennie, D.; Moher, D.; Becker, B.J.; Sipe, T.A.; Thacker, S.B.; et al. Meta-analysis of observational studies in epidemiology: A proposal for reporting. JAMA 2000, 283, 2008-2012. [CrossRef] [PubMed]

46. Shin, I.-S.; Rim, C.H. Updating Perspectives on Meta-Analyses in the Field of Radiation Oncology. Medicina 2021, 57, 117. [CrossRef] [PubMed]

47. Shrier, I.; Boivin, J.-F.; Steele, R.J.; Platt, R.W.; Furlan, A.; Kakuma, R.; Brophy, J.; Rossignol, M. Should meta-analyses of interventions include observational studies in addition to randomized controlled trials? A critical examination of underlying principles. Am. J. Epidemiol. 2007, 166, 1203-1209. [CrossRef]

48. Vandenbroucke, J.P. When are observational studies as credible as randomised trials? Lancet 2004, 363, 1728-1731. [CrossRef] 